\title{
Ionisation of Atomic Hydrogen by Positron Impact
}

\author{
Gottfried Spicher, Björn Olsson, Wilhelm Raith, Günther Sinapius \\ and Wolfgang Sperber \\ Fakultät für Physik, Universität Bielefeld \\ D-4800 Bielefeld 1, Federal Republic of Germany
}

\section{$1 \quad$ Experimental Setup}

With our crossed beam apparatus [1] we measure the relative impact-ionisation cross section of atomic hydrogen by positron impact. A layout of the scattering region is given in fig. 1 .

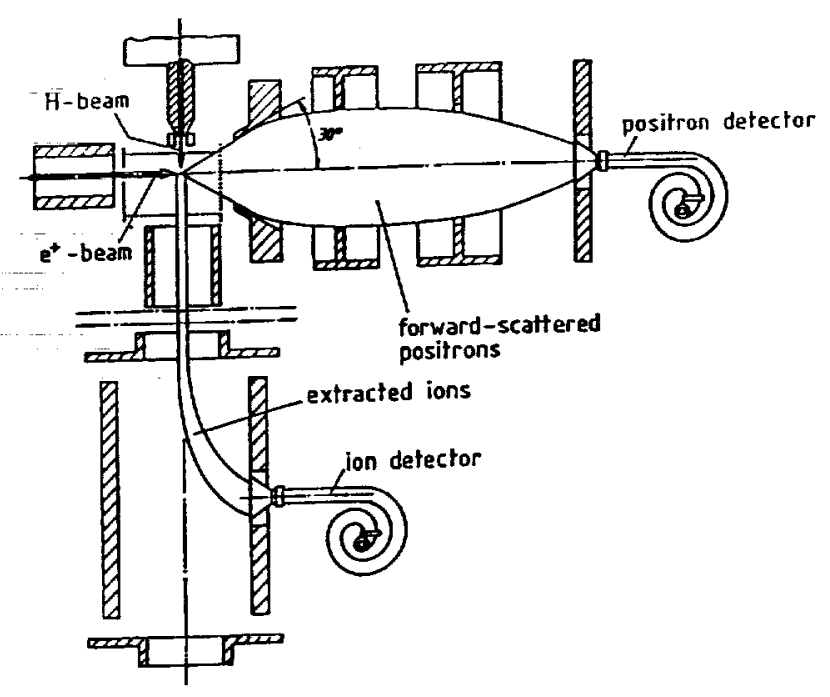

Figure 1: Layout of the experiment

\section{Data Taking}

Because of the $\mathrm{H}_{2}$-molecules in the atomic beam and the residual gas both $\mathrm{H}_{1}^{+}$and $\mathrm{H}_{2}^{+}$ions are produced (see table 1). Positrons ionize atoms or molecules through two different processes: (a) impact ionisation: leaving an ion, a free electron and the projectile, or (b) positronium formation: leaving an ion and a positronium 'atom'. Impact ionisation leads to time-correlated signals on both detectors. The positron and the ion signals are processed by a time- to-pulse-height converter. If the ion follows the projectile in less than $4 \mu \mathrm{s}$ this event is stored in a multichannel analyzer, $\mathrm{H}_{1}^{+}$and $\mathrm{H}_{2}^{+}$ions can be distinguished by there different flight times. Because of a relative high background on the ion-detector $\left(\sim 10 \mathrm{~s}^{-1}\right)$ produced by $\mathrm{L}_{\alpha}$-photons we analyze timecorrelated signals only. In our set-up the detection of atomic ions produced via dissociative ionisation plays a minor role (less than $1 \%$ of the total ionisation signals). As long as the detection probabilities for the correlated positron-ion pairs and the overlapp of projectile and target beam are unknown we can only determine relative ion-formation probabilities. By switching the polarity of the optical elements for the primary beam transport we can also measure the respective values for electron impact. To obtain $\sigma_{I o n}^{-}\left(H_{1}\right)$ and $\sigma_{I o n}^{-}\left(H_{2}\right)$ the ion-formation probabilities are normalized at $100 \mathrm{eV}$ to the data of Shah et al. [2] and Rapp, Englander-Golden [3], respectively. The same normalization factors are also used for the normalization of the positron impact ionisation data on $\mathrm{H}_{1}$ and $\mathrm{H}_{2}$, respectively. Fortunately we can check this procedure by comparing our $\mathrm{e}^{+}-\mathrm{H}_{2}$ results with those obtained in a different apparatus [4]. The energy of the projectiles can be variied between $10 \mathrm{eV}$ and $600 \mathrm{eV}$; the intensities are in the order of $3000 \mathrm{~s}^{-1}$. The observed ion-formation probabilities are rather low ( $\leq 5 \times 10^{-6}$ ions/projectile), so automated around-the-clock measurements were performed for more than 100 days to obtain the presented data.

\section{Results}

In figure 2 the first measurements on the ionisation of atomic hydrogen by positron impact are shown. 


\begin{tabular}{||l|l|r||}
\hline \hline Process & Cross Section & Threshold \\
\hline \hline $\mathrm{e}^{+}+\mathrm{H}_{1} \rightarrow \mathrm{Ps}+\mathrm{H}_{1}^{+}$ & $\sigma_{P s}\left(H_{1}\right)$ & $6.8 \mathrm{eV}$ \\
$\mathrm{e}^{+}+\mathrm{H}_{1} \rightarrow \mathrm{e}^{+}+\mathrm{e}^{-}+\mathrm{H}_{1}^{+}$ & $\sigma_{\text {Ion }}\left(H_{1}\right)$ & $13.6 \mathrm{eV}$ \\
\hline $\mathrm{e}^{+}+\mathrm{H}_{2} \rightarrow \mathrm{Ps}+\mathrm{H}_{2}^{+}$ & $\sigma_{P s}\left(H_{2}\right)$ & $8.6 \mathrm{eV}$ \\
$\mathrm{e}^{+}+\mathrm{H}_{2} \rightarrow \mathrm{e}^{+}+\mathrm{e}^{-}+\mathrm{H}_{2}^{+}$ & $\sigma_{\text {Ion }}\left(H_{2}\right)$ & $15.4 \mathrm{eV}$ \\
\hline $\mathrm{e}^{+}+\mathrm{H}_{2} \rightarrow \mathrm{Ps}_{1}+\mathrm{H}_{1}+\mathrm{H}_{1}^{+}$ & $\sigma_{P s, D i s s}\left(H_{2}\right)$ & $11.1 \mathrm{eV}$ \\
$\mathrm{e}^{+}+\mathrm{H}_{2} \rightarrow \mathrm{e}^{+}+\mathrm{e}^{-}+\mathrm{H}_{1}+\mathrm{H}_{1}^{+}$ & $\sigma_{\text {Ion, Diss }}\left(H_{2}\right)$ & $17.9 \mathrm{eV}$ \\
\hline
\end{tabular}

Table 1: The most important processes for the positron impact ionisation of atomic and molecular hydrogen

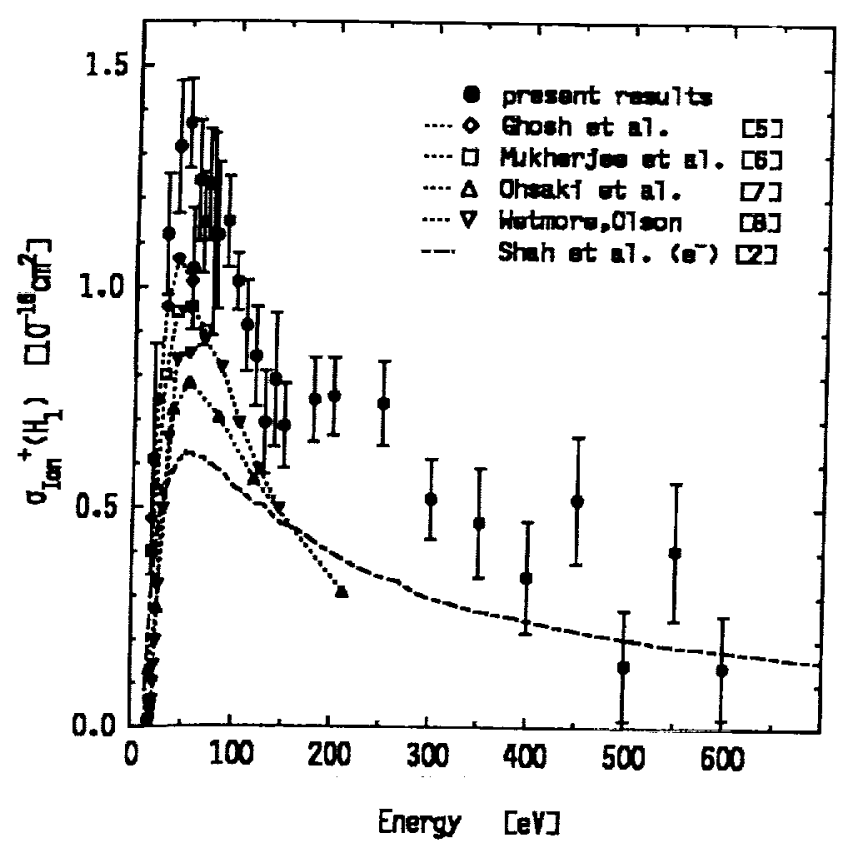

Figure 2: Positron impact ionisation cross sections: I. $\sigma_{\text {Ion }}^{+}\left(H_{1}\right)$.

Below $400 \mathrm{eV}$ the $\mathrm{e}^{+}-\mathrm{H}_{1}$ impact ionisation cross section (fig. 2) is significantly higher than the respective $\mathrm{e}^{-}$cross section, at $50 \mathrm{eV}$ about a factor of two. Qualitatevely the cross section shows the shape predicted by the theoretical estimates, but at maximum all calculated values are too low. As mentioned above we measure the positron impact ionisation cross section for molecular hydrogen simultaneously (fig. 3). Our values agree excellently with those from Fromme et al. [4]. In order to check the performance of the apparatus we measure the number of time-correlated $\mathrm{H}_{1}^{+}$- and $\mathrm{H}_{2}^{+}$-ions for electron impact ionisation. The comparisons (fig. 4,5) show a good agreement.

Note: We detect only those projectiles that are scattered into a angular sphere of $\pm 30^{\circ}$. This may cause two errors in the detection of the correlated

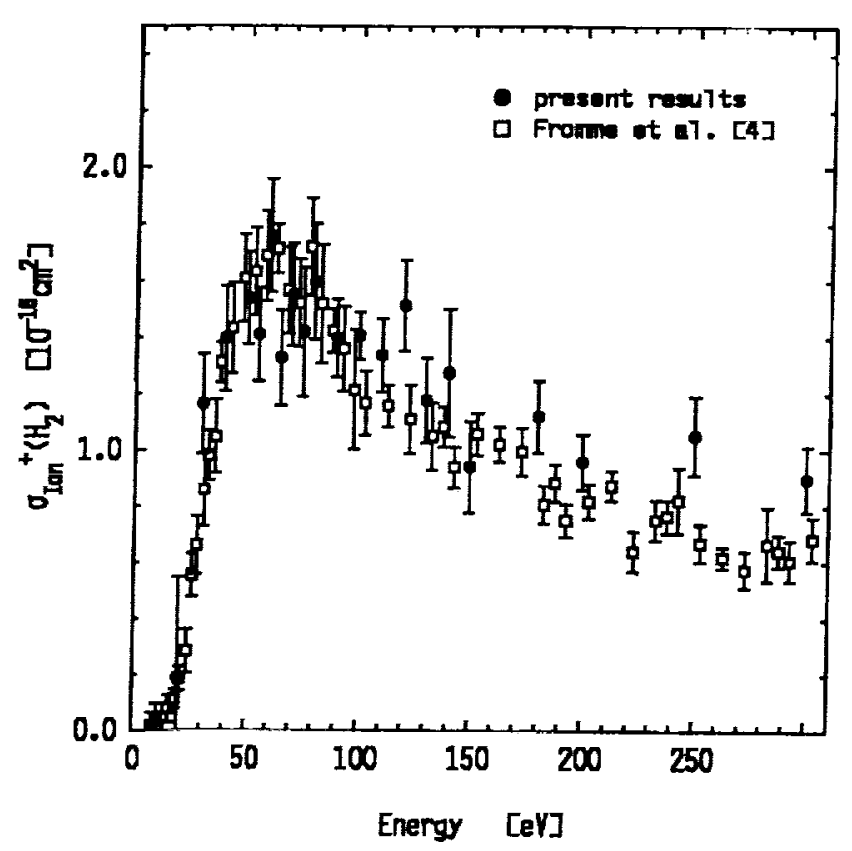

Figure 3: Positron impact ionisation cross sections: II. $\sigma_{\text {Ion }}^{+}\left(H_{2}\right)$.

ion-projectile-pairs, depending on (a) the energy or, (b) the charge of the scattering projectile. To correct for the effect of error (a) we will form the ratio of the ion-formation probabilities produced by $\mathrm{e}^{+}$and $\mathrm{e}^{-}$ and multiplied them with the $\mathrm{e}^{-}$cross sections from literature. So far there is only incomplete information on the effect of error (b).

\section{Future / Acknowledgements}

In a collaboration of members of the Brookhaven $\mathrm{Na}$ tional Laboratory, the City College of CUNY and the University of Bielefeld this experiment will be continuied at BNL. The apparatus will be modified and it will be possible to achieve more precise data with higher positron intensities, especially at ener- 


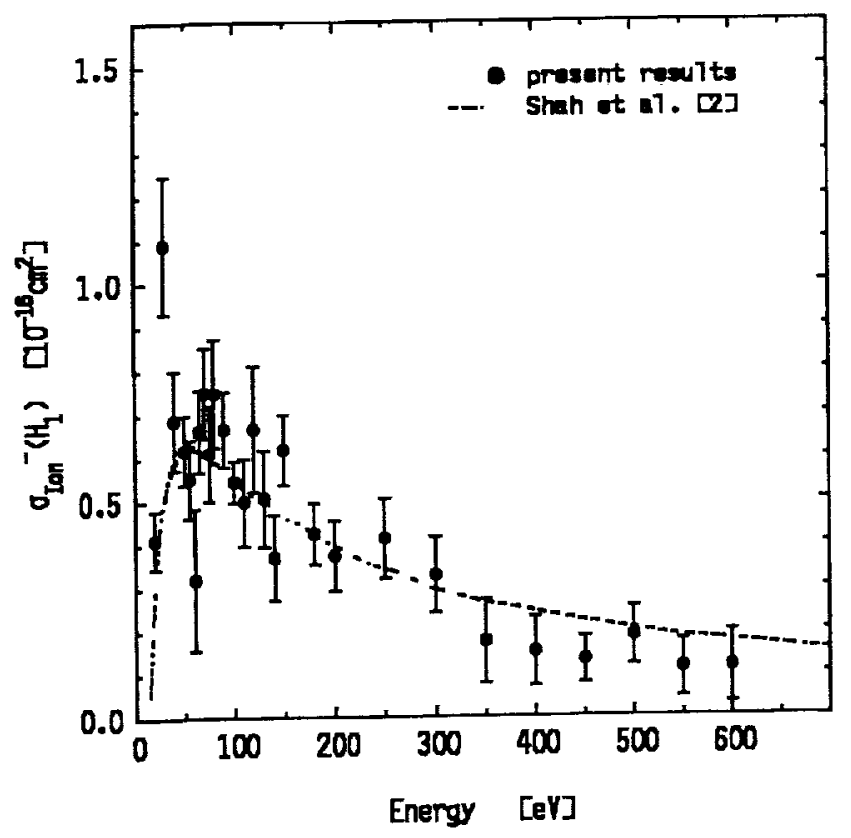

Figure 4: Electron impact ionisation cross sections: I. $\sigma_{\text {Ion }}^{-}\left(H_{1}\right)$.

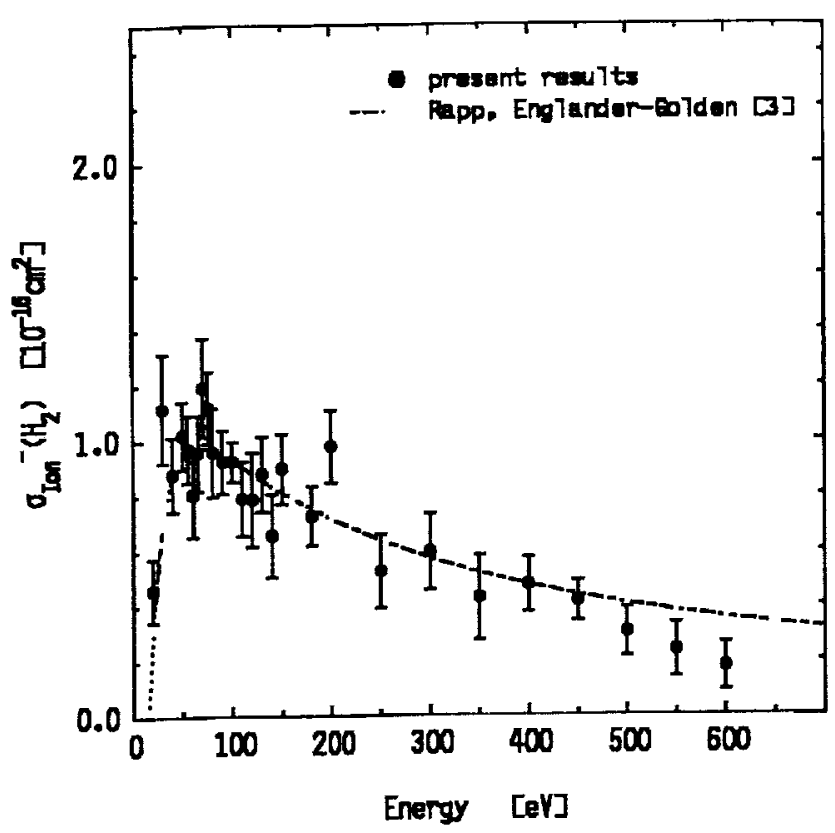

Figure 5: Electron impact ionisation cross sections: II. $\sigma_{\text {Ion }}^{-}\left(H_{2}\right)$. gies near the threshold. The work at Bielefeld was supported by the Deutsche Forschungsgemeinschaft (DFG). Our Brookhaven project is being supported by the Bundesministerium für Forschung und Technologie (BMFT).

\section{References}

[1] Spicher, G., Gläsker, A., Raith, W., Sinapius, G., Sperber, W.

in: Humberston, J.W., Armour, E.A.G. [eds.]

Atomic Physics with Positrons

New York, 1987 (NATO ASI series. Series B, Physics; vol. 101)

[2] Shah, M.B., Elliot, D.S., Gilbody, H.B.

J. Phys. B: At. Mol. Phys. 20 (1987), 3501 3514

[3] Rapp, D., Englander-Golden, P.

J. Chem. Phys. 43 (1965), 1464 - 1479

[4] Fromme, D., Kruse, G., Raith, W., Sinapius, G.

J. Phys. B: At. Mol. Phys. $\underline{21}$ (1988), L261 L265

[5] Ghosh, A.S., Majumdar, P.S., Basu, M. Can. J. Phys. $\underline{63}$ (1985), $621-624$

[6] Mukherjee, K.K., Singh, N.R., Mazumdar, P.S. J. Phys. B: At. Mol. Phys. 222 (1989), 99 - 103

[7] Ohsaki, A., Watanabe, T., Nakanishi, K., Iguchi, K.

Phys. Rev. A 32 (1985), 2640 - 2644

[8] Wetmore, A.E., Olson, R.E.

Phys. Rev. $\underline{34}$ (1986), $2822-2829$ 
